\title{
Combustibles alternativos para motores de combustión interna obtenidos a partir de residuos plásticos
}

\section{Alternative fuels for internal combustion engines obtained from plastic waste}

\author{
RIESCO-ÁVILA, José Manuel†*, RODRÍGUEZ-VALDERRAMA, David Alejandro, PARDO-CELY, \\ Diana Marcela y ELIZALDE- BLANCAS, Francisco
}

Universidad de Guanajuato, Departamento de Ingeniería Mecánica, División de Ingenierías Campus Irapuato-Salamanca

ID $1^{\text {er }}$ Autor: José Manuel, Riesco-Ávila / ORC ID: 0000-0001-7810-2566, CVU CONACYT ID: 120186

ID $1^{\text {er }}$ Coautor: David Alejandro, Rodríguez-Valderrama / ORC ID: 0000-0002-7786-0653, CVU CONACYT ID: 792656

ID $2^{\text {do }}$ Coautor: Diana Marcela, Pardo-Cely / ORC ID: 0000-0002-6178-1096, CVU CONACYT ID: 791608

ID $3^{\text {er }}$ Coautor: Francisco, Elizalde- Blancas / ORC ID: 0000-0002-2372-949X, CVU CONACYT ID: 43798

DOI: $10.35429 / J C P E .2019 .20 .6 .22 .29$

Recibido 09 Junio, 2019; Aceptado 28 Septiembre, 2019

\section{Resumen}

De los diferentes métodos existentes para el reciclaje de plástico, la pirólisis ofrece la posibilidad de resolver las limitaciones del reciclado mecánico, el cual necesita grandes cantidades de residuos plásticos limpios, separados y homogéneos para poder garantizar la calidad del producto final. La pirólisis consiste en la descomposición química de los materiales plásticos por degradación térmica en ausencia de oxígeno. Los residuos plásticos son introducidos en una cámara, donde son sometidos a altas temperaturas y los gases generados se condensan con el fin de obtener un destilado de hidrocarburos. En este trabajo se presentan los resultados obtenidos de la pirólisis de mezclas de residuos plásticos de polipropileno, polietileno de alta densidad y polietileno de baja densidad. En una primera etapa, los residuos son sometidos a un proceso de pirólisis rápida a temperaturas de $440-450^{\circ} \mathrm{C}$, obteniéndose una mezcla de hidrocarburos pesados. Posteriormente, estos hidrocarburos son sometidos a un proceso de destilación, primero a una temperatura de $180^{\circ} \mathrm{C}$, donde se obtiene un hidrocarburo con propiedades similares a las de la gasolina, y después a una temperatura de $360^{\circ} \mathrm{C}$, obteniéndose un hidrocarburo con propiedades similares a las del diesel.

Pirólisis, Residuos Plásticos, Hidrocarburos

\begin{abstract}
Of the different methods for recycling plastic, pyrolysis offers the possibility to overcome the limitations of mechanical recycling, which requires large amounts of clean, separate and homogeneous plastic waste to ensure the quality of the final product. Pyrolysis is the chemical decomposition of plastic materials by thermal degradation in the absence of oxygen. The plastic waste is introduced into a chamber, where it is subjected to high temperatures, and the gases generated are condensed in order to obtain a distillate hydrocarbon. This paper presents the results obtained from the pyrolysis of plastic waste mixtures of polypropylene, high density polyethylene, and low density polyethylene. In a first stage, the plastic waste is subjected to a rapid pyrolysis process at temperatures of $440-450{ }^{\circ} \mathrm{C}$, obtaining a mixture of heavy hydrocarbons. Subsequently, these hydrocarbons are subjected to a distillation process, first at a temperature of $180^{\circ} \mathrm{C}$, where a hydrocarbon with properties similar to those of gasoline is obtained, and then at a temperature of $360{ }^{\circ} \mathrm{C}$, yielding a hydrocarbon with properties similar to those of diesel.
\end{abstract}

Pyrolysis, Plastic Waste, Hydrocarbons

Citación: RIESCO-ÁVILA, José Manuel, RODRÍGUEZ-VALDERRAMA, David Alejandro, PARDO-CELY, Diana Marcela y ELIZALDE- BLANCAS, Francisco. Combustibles alternativos para motores de combustión interna obtenidos a partir de residuos plásticos. Revista de Energía Química y Física. 2019. 6-20: 20-27

\footnotetext{
* Correspondencia al Autor (Correo electrónico: riesco@ugto.mx)

$\dagger$ Investigador contribuyendo como primer Autor
} 


\section{Introducción}

El plástico es uno de los grandes inventos del siglo XX, es un material ligero, flexible, versátil y barato, por lo que es utilizado para la fabricación de una infinidad de productos en todo tipo de sectores (hogar, comercio, industria, agricultura, construcción, automoción, etc.). Sin embargo, el incremento en el uso de estos productos en los últimos años está provocando que cada vez haya más residuos plásticos, lo cual, aunado a su lenta degradación, representa una grave amenaza para el medio ambiente.

El reciclaje de plástico ha recibido mucha atención y se han desarrollado muchas técnicas para mejorarlo. Algunos de estos procedimientos empezaron a desarrollarse en los años 70's, cuando algunos países comenzaron a incinerar sus residuos plásticos. Desde entonces, ha habido muchos avances en la manera de reciclar plásticos, dando como resultado cuatro tipos de reciclaje: primario, secundario, terciario $\mathrm{y}$ cuaternario (Kumar Panda, 2011).

El proceso de reciclaje primario es fundamentalmente el mismo para los distintos plásticos. Consiste en la separación, limpieza, peletizado, moldeado, moldeado por inyección, moldeado por compresión y termoformación. Este proceso produce una materia prima de alta calidad, similar a las del polímero original. El reciclaje secundario difiere del primario únicamente en que aquí no hay separación de los plásticos a reciclar, por lo que los productos obtenidos son de propiedades inferiores a las del polímero original. Este método elimina la necesidad de separar y limpiar y de esa forma la mezcla de plásticos (incluyendo tapas de aluminio, etiquetas de papel, polvo, etc.), se muele y funde dentro de un extrusor.

El reciclaje terciario degrada al polímero a compuestos químicos básicos y combustibles. Este tipo de reciclaje es diferente de los dos primeros, fundamentalmente porque involucra un cambio químico, no sólo un cambio físico. En el reciclaje terciario las largas cadenas del polímero se rompen en pequeños hidrocarburos (monómeros) o monóxido de carbono e hidrógeno. Actualmente, el reciclaje terciario cuenta con dos métodos principales: pirólisis y gasificación, no obstante, se están desarrollando otros métodos como la metanólisis y la glicólisis.
La metanólisis es la ruptura de las cadenas causada por metanol y la glicólisis es la ruptura de un enlace glicosídico producida por alguna sustancia. (Rezagos.com, 2019). El reciclaje cuaternario consiste en el calentamiento del plástico con el objeto de usar la energía térmica liberada de este proceso, para llevar a cabo otros procedimientos; por ejemplo, (Ilie Ionut, 2019) realizó un diseño para obtener energía eléctrica a partir de combustibles sólidos recuperados.

La incineración puede incluirse en esta clasificación siempre que la recuperación de energía térmica sea acompañada de un generador de vapor o por el uso directo de gases de alta temperatura, en un proceso que requiera una fuente de calor externa. La incineración posee algunas ventajas: (a) disminuye considerablemente la cantidad de espacio ocupado en los rellenos sanitarios, (b) la recuperación de metales y (c) el manejo de diferentes cantidades de desechos. Sin embargo, algunas de sus desventajas son: la generación de contaminantes gaseosos y la gran inversión monetaria que representa.

De los diferentes métodos descritos anteriormente, la pirólisis ofrece la posibilidad de resolver las limitaciones del reciclado mecánico (primario y secundario), el cual necesita grandes cantidades de residuos plásticos limpios, separados y homogéneos para poder garantizar la calidad del producto final. En la pirólisis, no es necesaria la clasificación ni limpieza de los distintos tipos de residuos plásticos y es posible procesar residuos contaminados con alimentos y productos químicos, como insecticidas, herbicidas y fertilizantes, reduciendo costos de clasificación y limpieza.

La pirólisis consiste en la descomposición química de los materiales plásticos por degradación térmica en ausencia de oxígeno. Los residuos plásticos son introducidos en una cámara, donde son sometidos a altas temperaturas y los gases generados se condensan con el fin de obtener un destilado de hidrocarburos.

El objetivo de este trabajo es obtener combustibles mediante la pirólisis de mezclas arbitrarias de residuos plásticos, de polipropileno, polietileno de alta densidad y polietileno de baja densidad. 
Para esto, en una primera etapa, los residuos son sometidos a un proceso de pirólisis rápida y posteriormente, los productos obtenidos son sometidos a un proceso de destilación, primero a una temperatura de $180{ }^{\circ} \mathrm{C}$ y después a una temperatura de $360{ }^{\circ} \mathrm{C}$. Los productos obtenidos son caracterizados para obtener sus propiedades fisicoquímicas.

En la sección 2, se muestra la metodología usada para realizar el proceso de pirólisis, además de los materiales y instrumentos usados, para la medición de las propiedades. En la sección 3, se muestran los resultados del proceso de pirólisis de la mezcla de plásticos, de donde se obtiene una mezcla de hidrocarburos pesados, y los resultados del proceso de destilación, de donde se obtienen combustibles con propiedades similares a la gasolina y el diesel.

\section{Metodología}

El material usado en este trabajo es una mezcla de residuos plásticos posconsumo en proporciones arbitrarias de polipropileno (PP), polietileno de alta densidad (HDPE) y polietileno de baja densidad (LDPE), el material se muestra en la Figura 1. Este material fue pirolizado en un reactor por lotes (batch) de acero inoxidable, aislado térmicamente, que contiene dos resistencias eléctricas controladas mediante dos termopares tipo $\mathrm{K}$, los cuales muestran la temperatura dentro del reactor en la parte inferior y superior del mismo.

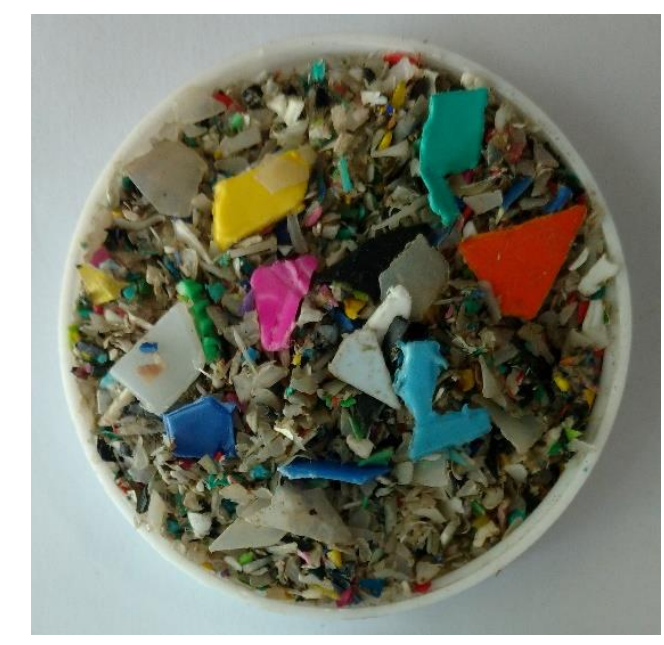

Figura 1 Mezcla de residuos plásticos.

La mezcla de residuos plásticos se introduce al reactor y luego se sella herméticamente para iniciar el proceso de calentamiento.
Conforme la temperatura se incrementa en el reactor, el aire contenido en el mismo es desalojado a través de un tubo que desemboca en un baño de agua a temperatura ambiente, el cual sirve de sello hidráulico, debido a esto se impide que el aire se devuelva nuevamente al reactor produciendo así una atmósfera inerte dentro del mismo. Cuando se alcanzan las temperaturas de evaporación de la mezcla de residuos plásticos y en ausencia de oxígeno, se produce el proceso de pirólisis. Los gases producidos son desalojados hacia el baño de agua donde se condensan. Debido a la menor densidad de los productos de la pirólisis condensados, éstos se separan del agua y de ahí se recuperan a través de una válvula de desagüe.

El reactor también incluye un filtro de carbón activado, por donde son desalojados los gases no condensables que se producen en la pirólisis y una válvula para el drenado del agua utilizada para la condensación del producto líquido. En la Figura 2 se presenta el diagrama esquemático de este reactor.

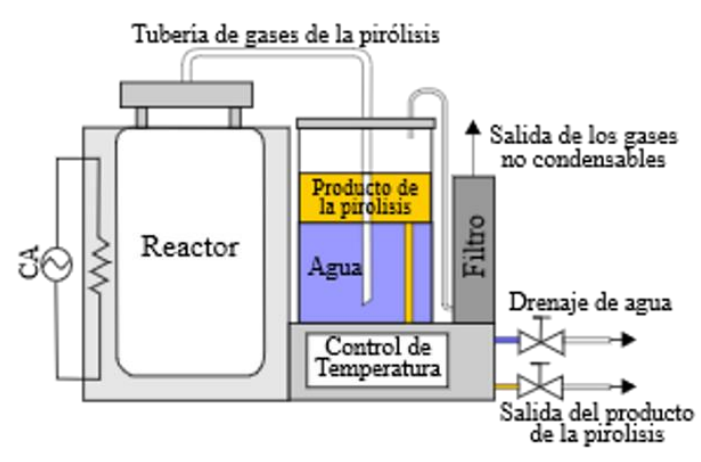

Figura 2 Pirolizador

Se caracterizaron las muestras de los productos obtenidos de la pirólisis en estado líquido, para obtener sus propiedades fisicoquímicas, para esto, se usó un cromatógrafo de gases Varian CG-450 (Figura 3a), dotado de un software especial que permite identificar hasta 133 hidrocarburos. Para la medición de la densidad se usó un picnómetro de $50 \mathrm{~mL}$ con una incertidumbre de $\pm 0,0015$ (Figura 3b), la medición se hizo de acuerdo a lo especificado en la norma ISO 3838 (2004). La viscosidad cinemática se obtuvo con un viscosímetro Cannon-Fenske (Figura 3c), en el cual se midió el tiempo necesario para que el volumen de líquido fluyera por gravedad a través de un tubo capilar estándar a $40^{\circ} \mathrm{C}$. Esta última propiedad se midió como lo especifica la norma ASTM D 445 (2015). 

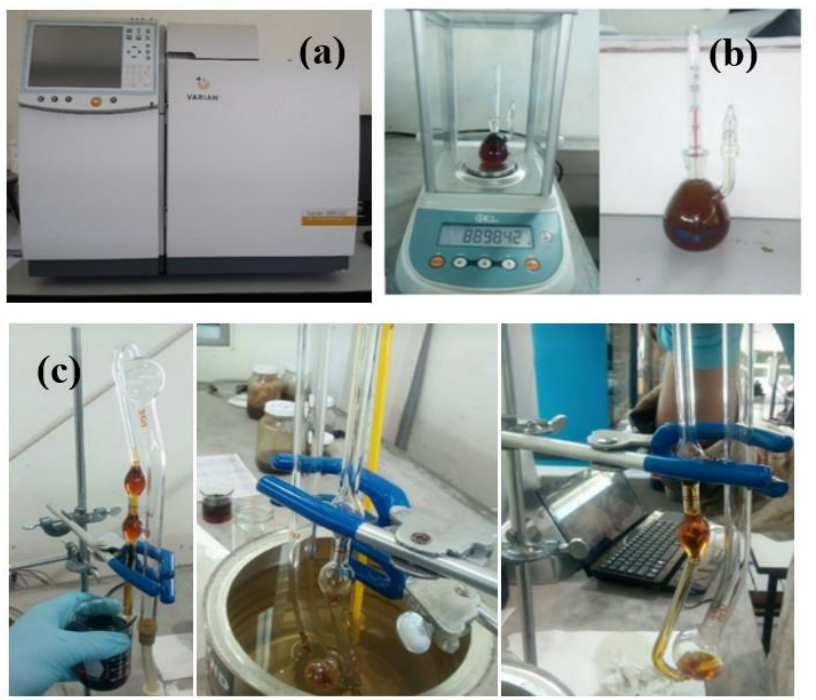

Figura 3 Equipos de medición, (a) Cromatógrafo de gases, (b) Balanza Analítica y Picnómetro, (c) Viscosímetro Cannon-Fenske

\section{Resultados}

\section{Pirólisis}

La primera etapa de este estudio consistió en realizar el proceso de pirólisis para la mezcla de residuos plásticos. Se realizaron dos pruebas, y sus réplicas, cada una con un kilogramo de residuos plásticos, con el fin de encontrar la temperatura en que se presenta la mejor relación de conversión entre la mezcla y el producto de pirólisis. La primera prueba se realizó a una temperatura de $440{ }^{\circ} \mathrm{C}$ y la segunda prueba a una temperatura de $460^{\circ} \mathrm{C}$. Los productos obtenidos se muestran en la Figura 4.

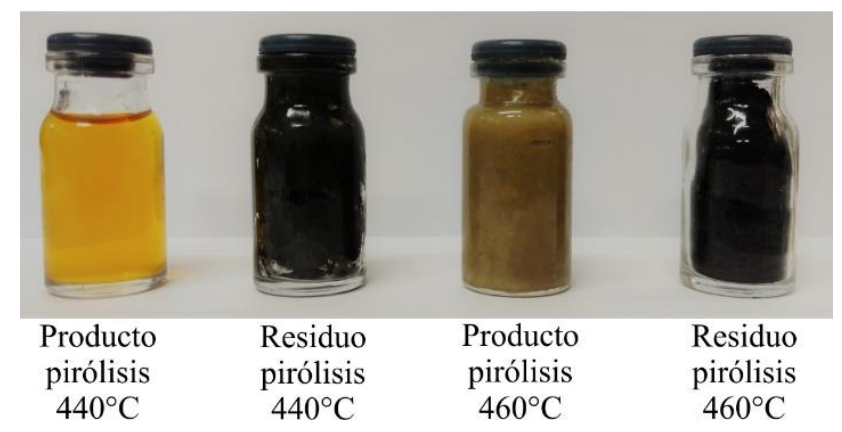

Figura 4 Productos de la pirólisis

El Gráfico 1 muestra los porcentajes promedio de la masa de productos recuperados por medio de la pirólisis a temperaturas constante. En la pirólisis a $440{ }^{\circ} \mathrm{C}$ se recuperó un $61 \%$ en producto líquido, un $21 \%$ de residuos en forma de cera y el $18 \%$ restante son gases no condensables.
El producto de la pirólisis de residuos plásticos a temperatura de $460{ }^{\circ} \mathrm{C}$ fue principalmente cera, con un porcentaje de recuperación del $81 \%$, en promedio se produjeron $2.5 \%$ de residuos en forma de ceniza y el $16.5 \%$ en forma de gases no condensables.

Es importante indicar que la cera resultante de la pirolisis a $440{ }^{\circ} \mathrm{C}$, es un producto que contiene una cantidad de polímeros sin degradar y es posible recuperarlo con temperaturas superiores para obtener un producto con características similares al obtenido a la temperatura de $460{ }^{\circ} \mathrm{C}$.

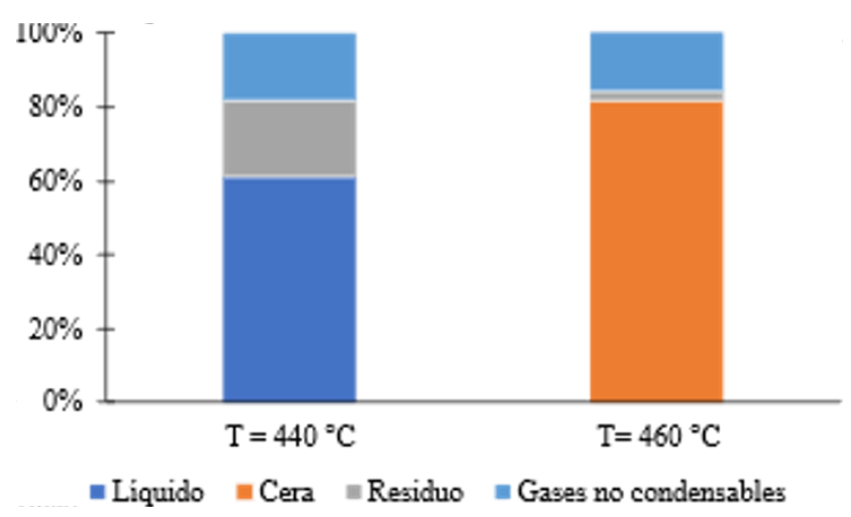

Gráfico 1 Porcentaje en masa de los productos obtenidos de la pirólisis a temperaturas de $400{ }^{\circ} \mathrm{C}$ y $460{ }^{\circ} \mathrm{C}$

En comparación con los resultados presentes en la literatura para procesos de pirólisis de un solo polímero se presentan algunas similitudes en cuanto a la temperatura de degradación. Por ejemplo, Marcilla et al. (2005) encontraron que a la temperatura de $467^{\circ} \mathrm{C}$ se produjo la máxima de degradación para el polietileno de alta densidad y para el polipropileno se produjo a los $447^{\circ} \mathrm{C}$. Para el polietileno de baja densidad Marcilla et al. (2009) encontraron que el rendimiento máximo de producto líquido se produjo a temperaturas superiores a $460^{\circ} \mathrm{C}$.

También, Jung et al. (2010) reportan que la temperatura de descomposición para el polietileno y el polipropileno se encuentra en el rango de 400 a $500{ }^{\circ} \mathrm{C}$. En cuanto a los productos obtenidos de la pirólisis de mezclas, Jung et al. (2010) muestran que se puede obtener un rendimiento máximo del $60 \%$ en peso, para la mezcla de polietileno y polipropileno, obteniendo un producto líquido. 
Donaj et al. (2012) realizaron el proceso de pirólisis térmica y catalítica para una mezcla de polietilenos y polipropileno, obteniendo un porcentaje de producto líquido de $48.4 \%$ en peso para la pirólisis térmica y del $89 \%$ en peso para la pirólisis catalítica, los dos procesos a una temperatura de $500{ }^{\circ} \mathrm{C}$. Adicionalmente, Cajas, B, \& Campaña, F, (2019), encontraron que se puede obtener un rendimiento de hasta el 94.33 $\%$ de combustible líquido a partir de la mezcla de polímeros.

\section{Destilaciones}

Con el propósito de mejorar el producto de las pirólisis (líquido o cera) se realizaron destilaciones fraccionadas a diferentes temperaturas y obtener así productos con propiedades similares a la gasolina y el diesel comerciales. Es importante indicar que los productos de las destilaciones de la cera o del producto líquido, presentaron propiedades y composiciones similares, por esta razón se habla de destilación del producto de la pirólisis.

Las destilaciones se hicieron en el reactor usado para el proceso de pirólisis y los productos obtenidos se muestran en la Figura 5. Inicialmente se realizó una destilación de los productos de pirólisis hasta $\operatorname{los} 360{ }^{\circ} \mathrm{C}$, debido a que hasta esta temperatura se obtienen productos líquidos y los productos que se obtienen a temperaturas superiores ya presentan formaciones de cera.

En la segunda etapa, se destiló el producto de la pirólisis a una temperatura de 180 ${ }^{\circ} \mathrm{C}$, de donde se obtuvo un producto de apariencia clara, luego se elevó la temperatura hasta los $360{ }^{\circ} \mathrm{C}$, para obtener la fracción de producto líquido restante.

Las temperaturas para de destilación se tomaron de acuerdo al Handbook of industrial hydrocarbon processes (2011), donde se menciona que la gasolina derivada de la destilación del petróleo se obtiene en el rango de $1{ }^{\circ} \mathrm{C}$ a $216^{\circ} \mathrm{C}$ y usualmente a temperatura de 180 ${ }^{\circ} \mathrm{C}$. En cuanto al diesel, este se obtiene de la destilación a temperaturas entre $\operatorname{los} 180^{\circ} \mathrm{C}$ y los $380{ }^{\circ} \mathrm{C}$.

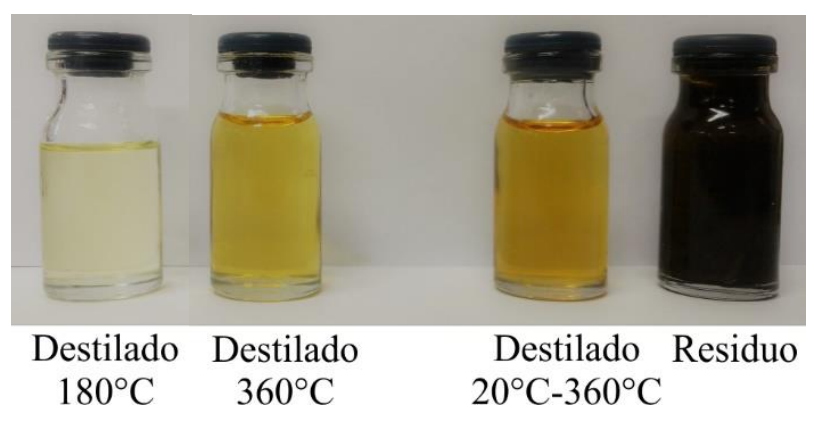

Figura 5 Productos de la destilación.

En el Grafico 2, se presenta el promedio de los porcentajes en masa de las destilaciones fraccionadas. En una mayor proporción se obtuvo un producto líquido que en promedio representa el $82 \%$ en masa, de los cuales el $10 \%$ corresponde al destilado a $180{ }^{\circ} \mathrm{C}$ y el $72 \%$ al destilado de 180 a $360{ }^{\circ} \mathrm{C}$. El restante $18 \%$ corresponde a los residuos en forma de cera (14\%) y a los gases no condensables (4\%).

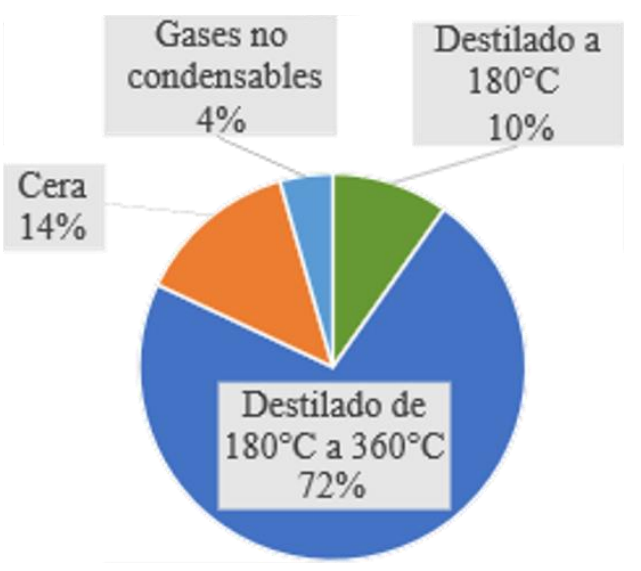

Gráfico 2 Porcentajes promedio de los productos destilados

En la Tabla 1 se muestran las propiedades medidas de los productos obtenidos durante el proceso de pirólisis y de destilación. Adicionalmente, se presentan las propiedades de la gasolina y del diesel comercial para su comparación. Los resultados muestran que la densidad relativa y la viscosidad del producto obtenido directamente de la pirólisis, son ligeramente menores que las del diesel. Por su parte, los valores de densidad relativa $\mathrm{y}$ viscosidad del destilado en una sola etapa $\left(20^{\circ} \mathrm{C}\right.$ a $360{ }^{\circ} \mathrm{C}$ ) son inferiores a las del diesel y superiores a las de la gasolina. Para el destilado a $180{ }^{\circ} \mathrm{C}$, los valores de densidad relativa $\mathrm{y}$ viscosidad se aproximan a los de la gasolina, mientras que los del destilado de $180{ }^{\circ} \mathrm{C}$ a 360 ${ }^{\circ} \mathrm{C}$ son semejantes a las del diesel. 
Los valores del poder calorífico (superior e inferior) son muy semejantes a las de los hidrocarburos comerciales, siendo ligeramente superiores los del destilado a $180{ }^{\circ} \mathrm{C}$.

\begin{tabular}{|c|c|c|c|c|}
\hline Sustancia & $\begin{array}{c}\text { Viscosidad } \\
\text { cinemática } \\
{\left[\mathrm{mm}^{2} / \mathrm{s}\right]} \\
@ 40^{\circ} \mathrm{C}\end{array}$ & $\begin{array}{c}\text { Densidad } \\
\text { relativa } \\
@ 20^{\circ} \mathrm{C}\end{array}$ & $\begin{array}{c}\text { Poder } \\
\text { calorífico } \\
\text { superior } \\
{[\mathbf{M J} / \mathbf{k g}]}\end{array}$ & $\begin{array}{c}\text { Poder } \\
\text { calorífico } \\
\text { inferior } \\
{[\mathrm{MJ} / \mathrm{kg}]}\end{array}$ \\
\hline Producto de Pirólisis & 3.060 & 0.791 & 45.584 & 43.815 \\
\hline $\begin{array}{l}\text { Destilado de } 20{ }^{\circ} \mathrm{C} \text { a } \\
360^{\circ} \mathrm{C}\end{array}$ & 2.288 & 0.779 & 45.918 & 43.856 \\
\hline Destilado a $180^{\circ} \mathrm{C}$ & 1.335 & 0.755 & 47.356 & 44.146 \\
\hline $\begin{array}{l}\text { Destilado de } 180^{\circ} \mathrm{C} \text { a } \\
360{ }^{\circ} \mathrm{C}\end{array}$ & 3.167 & 0.797 & 45.489 & 44.034 \\
\hline $\begin{array}{llr}\text { Gasolina } & \text { de } & 87 \\
\text { Octanos } & & \\
\end{array}$ & 1.050 & 0.749 & 45.544 & 42.813 \\
\hline Diesel & 3.620 & 0.831 & 44.595 & 43.463 \\
\hline
\end{tabular}

Tabla 1 Propiedades de los productos

\section{Cromatografía}

Con el fin de obtener la composición química de los productos obtenidos de la pirólisis y la destilación, se realizaron análisis por cromatografía de gases, estos resultados se muestran en el Gráfico 3. Por medio del estándar PIANO (Parafinas, Isoparafinas, Aromáticos, Naftenos y Oleofinas), se logró la identificación de algunas de las especies principales presentes en los productos líquidos de la prilólisis, los destilados y los combustibles comerciales.

El producto de la pirólisis (Gráfico 3a), el destilado de $20{ }^{\circ} \mathrm{C}$ a $360{ }^{\circ} \mathrm{C}$ (Gráfico $3 b$ ) y el destilado de $180^{\circ} \mathrm{C}$ a $360^{\circ} \mathrm{C}$ (Gráfico 3d) presentan similitud en su composición con el diesel (Gráfico 3f), con la diferencia de que contienen componentes con cadenas de carbón más pesados. Una característica común que presentan los productos destilados es que tienen un contenido alto de parafinas, con porcentajes mayores al $40 \%$, y porcentajes de elementos desconocidos cercanos al 50\%.

También sus componentes tienen una porción menor al $7 \%$ de cadenas de carbono desde los cinco hasta los 11 carbonos, y en su mayoría contienen cadenas de carbono de más de 11. El gran porcentaje de componentes desconocidos que se presta en las destilaciones hasta $360^{\circ} \mathrm{C}$, el producto de la pirólisis y el diesel, se debe a que los componentes principales tienen tiempo de elución muy altos que son cercanos o superiores al límite del estándar con el que se compararon y esta condición dificulta la caracterización de los componentes.
En cuanto a la composición del destilado a $180{ }^{\circ} \mathrm{C}$, este tiene algunas diferencias con respecto a la gasolina. Al observar el Gráfico 3, se puede notar que el destilado presenta componentes durante todo el tiempo de elución y la gasolina los presenta antes de los 40 minutos. En relación con la composición, la gasolina tiene el $20 \%$ de naftas y el destilado tan solo un $2.67 \%$. Con respecto a los aromáticos la gasolina cuenta con un $9.91 \%$ y el destilado con tan solo 2.98\%. En comparación con las parafinas el destilado presenta un mayor porcentaje con un $89.61 \%$ y la gasolina con $73.78 \%$; por lo tanto, se podría concluir que temperatura de $180{ }^{\circ} \mathrm{C}$ no es adecuada para la obtención de un hidrocarburo parecido en composición a la gasolina, debería ser una temperatura menor.

Se puede observar que el proceso de destilación permite separar los componentes con cadenas de carbono muy largas. Partiendo del producto de la pirólisis, se nota que la destilación a una temperatura de $360{ }^{\circ} \mathrm{C}$ aún tiene componentes en todo el tiempo de elución, pero presenta componentes en menor proporción entre los 120 y 140 minutos. La separación del producto de la destilación entre las temperaturas de $180{ }^{\circ} \mathrm{C}$ permite que salgan los componentes más livianos, haciendo que el producto de la destilación entre las temperaturas de $180{ }^{\circ} \mathrm{C}$ a $360{ }^{\circ} \mathrm{C}$ sea el más parecido en composición al diesel.

En la literatura se encuentran algunos estudios en los que se obtienen productos con propiedades similares a los hidrocarburos comerciales; por ejemplo, Sarker et al. (2012) realizaron la pirolisis y la destilación para el polietileno de baja densidad y encontraron que por medio de la destilaciones se puede recuperar un $10 \%$ en hidrocarburos livianos y un $88 \%$ de hidrocarburos pesados. Buekens y Huang (1998) encontraron por medio de procesos catalíticos que se puede obtener el $31.5 \%$ de hidrocarburos ligeros, un $21 \%$ de productos con cadenas de carbono de 8 a 16 carbonos y un $42 \%$ de hidrocarburos pesados.

Al comprar con los productos obtenidos, se puede comprobar que el proceso de destilación permite obtener productos similares a los combustibles comerciales y que el proceso permite aprovechar más del $95 \%$ del material que se ingresa a destilar, ya sea en forma de combustibles alternativos o en forma de cera.

RIESCO-ÁVILA, José Manuel, RODRÍGUEZ-VALDERRAMA, David Alejandro, PARDO-CELY, Diana Marcela y ELIZALDE- BLANCAS, Francisco. Combustibles alternativos para motores de combustión interna obtenidos a partir de residuos plásticos. Revista de Energía Química y Física. 2019. 
a) Producto de la pirólisis

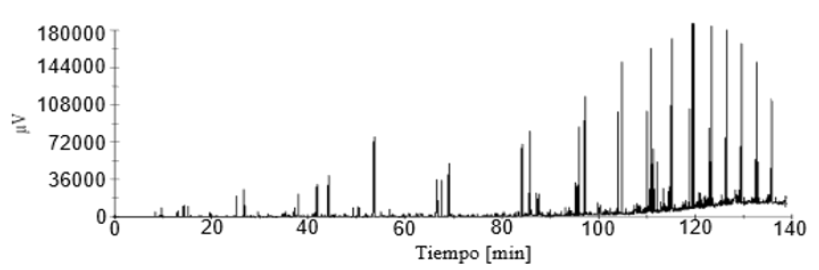

b) Destilado de $20^{\circ} \mathrm{C}$ a $360{ }^{\circ} \mathrm{C}$

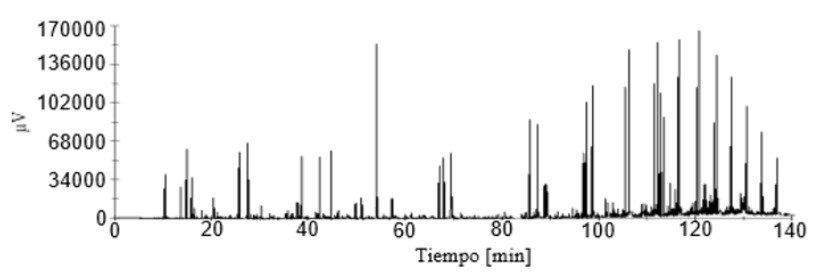

c) Destilado a $180^{\circ} \mathrm{C}$

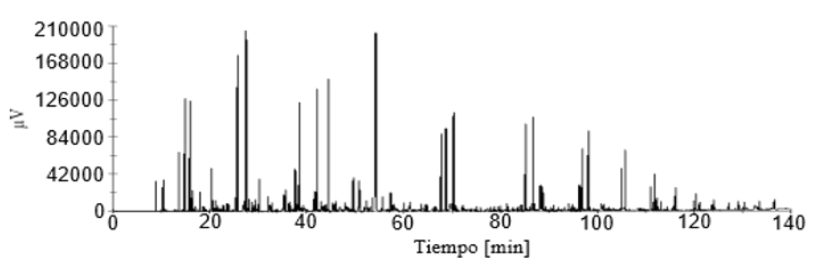

d) Destilado de $180{ }^{\circ} \mathrm{C}$ a $360{ }^{\circ} \mathrm{C}$

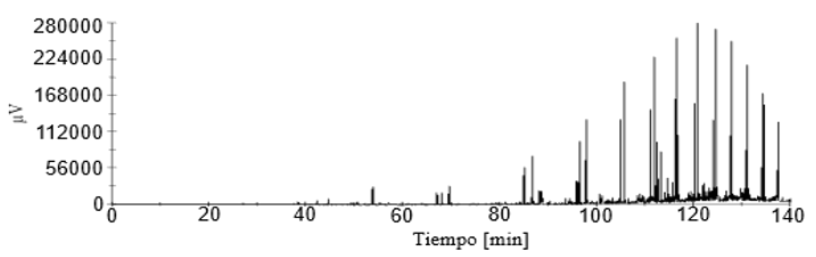

e) Gasolina de 87 Octanos

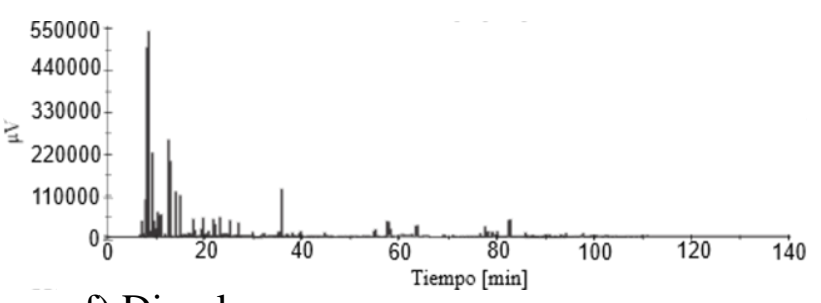

f) Diesel

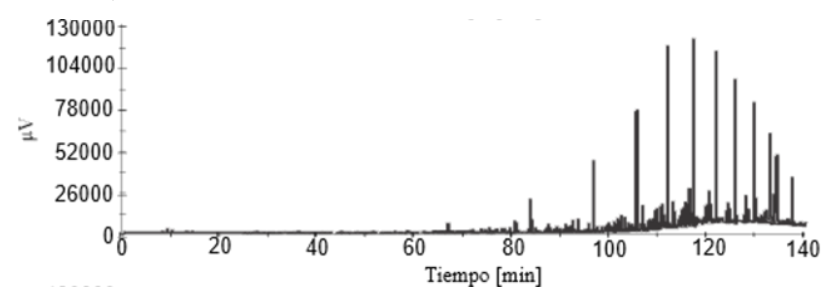

Gráfico 3 Cromatografías

\section{Conclusiones}

En este trabajo se presentan los resultados del proceso de pirólisis de una mezcla arbitraria de polímeros posconsumo y destilación del producto de la pirólisis. El proceso de pirólisis dio como resultado que se puede recuperar el $81 \%$ de la mezcla, en un producto que en sus propiedades de densidad relativa y viscosidad tiene similitudes al diesel.
El proceso de destilación fraccionada permitió la separación de una mezcla de hidrocarburos livianos, que tienen propiedades comparables con la gasolina. De igual forma, la destilación hasta una temperatura de $360{ }^{\circ} \mathrm{C}$ permitió obtener un producto con propiedades y composición similar al las del diesel comercial.

\section{Agradecimiento}

Los autores agradecen a la Secretaría de Innovación, Ciencia y Educación Superior del estado de Guanajuato (SICES), a la empresa RECICLA.LO, S.A. DE C.V. y a la Universidad de Guanajuato, el apoyo otorgado para la realización este proyecto.

\section{Referencias}

ASTM D445-15. (2015). Standard Test Method for Kinematic Viscosity of Transparent and Opaque Liquids (and Calculation of Dynamic Viscosity).

Buekens, A. G., \& Huang, H. (1998). Catalytic plastics cracking for recovery of gasoline-range hydrocarbons from municipal plastic wastes. Resources, Conservation and Recycling, 163 181.

Cajas Baque, A. M., \& Campaña Figueroa, B. L. (2019). Obtención y caracterizacion de un combustible obtenido a partir de los deschos del PET y Polipropileno (tesis de pregrado). Universidad de Guayaquil, Guayaquil, Ecuador. Donaj, P., Buzeto, F., Kaminsky, W., \& Yang, W. (2012). Pyrolysis of polyolefins for increasing the yield of monomers' recovery. Waste Management, 840-846.

Ilie Ionut, C. (2019). Diseño del proceso de gasificación para los combustibles sólidos recuperados procedentes de una planta de tratamiento mecánico biológico (tesis de maestría). Universidad Jaime I, España.

Jung, S.-H., Cho, M.-H., Kang, B.-S., \& Kim, J.S. (2010). Pyrolysis of a fraction of waste polypropylene and polyethylene for the recovery of BTX aromatics using a fluidized bed reactor. Fuel Processing Technology, 277-284 .

Kumar Panda, A. (2011). Studies on process optimization for production of liquid fuels from waste plastics. Roirkela. India: PhD. National Institute of Technology, Rourkela. India.

RIESCO-ÁVILA, José Manuel, RODRÍGUEZ-VALDERRAMA, David Alejandro, PARDO-CELY, Diana Marcela y ELIZALDE- BLANCAS, Francisco. Combustibles alternativos para motores de combustión interna obtenidos a partir de residuos plásticos. Revista de Energía Química y Física. 2019. 
Marcilla, A., Beltrán, M. I., \& Navarro, R. (2009). Evolution of products during the degradation of polyethylene in a batch reactor. Journal of Analytical and Applied Pyrolysis, 1421.

Marcilla, A., García-Quesada, J. C., Sánchez, S., \& Ruiz, R. (2005). Study of the catalytic pyrolysis behaviour of polyethylenepolypropylene mixtures. Journal of Analytical and Applied Pyrolysis, 387-392.

Rezagos.com. (2019, Jinio 26). Reciclado de plástico - Rezagos. Retrieved from http://www.rezagos.com/pages/reciclado-deplastico.html

Sarker, M., Rashid, M. M., Rahman, M. S., \& Molla, M. (2012). Fractional Distillation Process Utilized to Produce Light Fractional Fuel from Low Density Polyethylene (LDPE) Waste Plastic . The Open Fuels \& Energy Science Journal, 39-46.

Skoog, D. A., Holler, F. J., \& Nieman, T. A. (2001). Principios de analisis Instrumental. Aravaca (Madrid): Mc Graw-Hill / Interamericana de España.

Speight, J. G. (2011). Handbook of industrial hydrocarbon processes. Oxford, UK: Elsevier. UNE EN ISO3838. (2004). Crude Petroleum and Liquid or Solid Petroleum Products. Determination of Density or Relative Density. Capillary-Stoppered Pycnometerand Graduated Bicapillary Pycnometer Methods.
RIESCO-ÁVILA, José Manuel, RODRÍGUEZ-VALDERRAMA, David Alejandro, PARDO-CELY, Diana Marcela y ELIZALDE- BLANCAS, Francisco. Combustibles alternativos para motores de combustión interna obtenidos a partir de residuos plásticos. Revista de Energía Química y 